\title{
FINITE-DIMENSIONAL BANACH SPACES WITH NUMERICAL INDEX ZERO
}

\author{
MIGUEL MARTÍN, JAVIER MERÍ, AND ÁNGEL RODRÍGUEZ-PALACIOS
}

\begin{abstract}
We prove that a finite-dimensional Banach space $X$ has numerical index 0 if and only if it is the direct sum of a real space $X_{0}$ and nonzero complex spaces $X_{1}, \ldots, X_{n}$ in such a way that the equality$$
\left\|x_{0}+\mathrm{e}^{i q_{1} \rho} x_{1}+\cdots+\mathrm{e}^{i q_{n} \rho} x_{n}\right\|=\left\|x_{0}+\cdots+x_{n}\right\|
$$

holds for suitable positive integers $q_{1}, \ldots, q_{n}$, and every $\rho \in \mathbb{R}$ and every $x_{j} \in X_{j}(j=0,1, \ldots, n)$. If the dimension of $X$ is two, then the above result gives $X=\mathbb{C}$, whereas $\operatorname{dim}(X)=3$ implies that $X$ is an absolute sum of $\mathbb{R}$ and $\mathbb{C}$. We also give an example showing that, in general, the number of complex spaces cannot be reduced to one.
\end{abstract}

\section{INTRODUCTION}

The numerical index of a Banach space is a constant of the space relating the behaviour of the numerical radius with that of the usual norm on the Banach algebra of all bounded linear operators on the space. Let us recall the relevant definitions. Given a real or complex Banach space $X$, we write $B_{X}$ for the closed unit ball and $S_{X}$ for the unit sphere of $X$. The dual space will be denoted by $X^{*}$ and $L(X)$ will be the Banach algebra of all bounded linear operators on $X$.

The numerical range of an operator $T \in L(X)$ is the subset $V(T)$ of the scalar field defined by

$$
V(T)=\left\{x^{*}(T x): x \in S_{X}, x^{*} \in S_{X^{*}}, x^{*}(x)=1\right\} .
$$

This definition of numerical range was introduced by F. Bauer [1] as an extension of Toeplitz's numerical range of matrices [21], and, concerning applications, it is equivalent to Lumer's numerical range [12]. It is shown in [3, §9] that

$$
V(L(X), T):=\left\{\phi(T): \phi \in L(X)^{*},\|\phi\|=\phi(I d)=1\right\}=\overline{\mathrm{co}} V(T),
$$

where $\overline{\mathrm{co}}(A)$ denotes the closed convex hull of $A \subset X$ and $V(L(X), T)$ is the so-called algebra numerical range of $T$. The numerical radius of $T$ is given by

$$
v(T)=\sup \{|\lambda|: \lambda \in V(T)\} .
$$

It is clear that $v$ is a seminorm on $L(X)$ satisfying $v(T) \leqslant\|T\|$ for every $T \in L(X)$. Let us observe that the numerical radius of $T \in L(X)$ does not change if we replace $V(T)$ by $V(L(X), T)$. The numerical index of the space $X$ is defined as

$$
n(X)=\inf \left\{v(T): T \in S_{L(X)}\right\}
$$

Date: October 6th, 2003.

2000 Mathematics Subject Classification. 46B20, 47A12.

First author partially supported by Spanish MCYT projects BFM2000-1467 and BFM200200061. Second author supported by an FPU grant of the Spanish MECD. Third author partially supported by Junta de Andalucía grant FQM 0199. 
or, equivalently, as the greatest constant $k \geqslant 0$ such that $k\|T\| \leqslant v(T)$ for every $T \in L(X)$. Note that $0 \leqslant n(X) \leqslant 1$ and $n(X)>0$ if and only if $v$ and $\|\cdot\|$ are equivalent norms on $L(X)$. In the complex case, it is a celebrated result due to H. Bohnenblust and S. Karlin [2] (see also [8]) that $n(X) \geqslant 1 /$ e, so the numerical radius is always an equivalent norm. Classical references on this topic are the monographs by F. Bonsall and J. Duncan [3, 4]. For recent results we refer the reader to $[6,10,11,15,16,18]$ and the survey paper [13].

In this paper we deal with (real) Banach spaces with numerical index 0 . It is known that the class of such Banach spaces contains all Hilbert spaces of dimension greater than one, and all real spaces underlying complex Banach spaces (the operator $x \longmapsto i x$ on a complex Banach space has real numerical radius 0 ). One may think that Banach spaces with numerical index 0 have always some kind of "complex structure", but we will give later an example of an infinite-dimensional Banach space with numerical index 0 , not containing any isometric copy of $\mathbb{C}$. In the finite-dimensional context, such an example is not possible. Actually, the main result of this paper says that each finite-dimensional space $X$ with numerical index 0 is the direct sum of a real space $X_{0}$ (possibly equal to zero) and nonzero complex spaces $X_{1}, \ldots, X_{n}$ in such a way that the equality

$$
\left\|x_{0}+\mathrm{e}^{i q_{1} \rho} x_{1}+\cdots+\mathrm{e}^{i q_{n} \rho} x_{n}\right\|=\left\|x_{0}+\cdots+x_{n}\right\|
$$

holds for suitable positive integers $q_{1}, \ldots, q_{n}$, and every $\rho \in \mathbb{R}$ and every $x_{j} \in X_{j}$ $(j=0,1, \ldots, n)$. If the dimension of $X$ is two, then the above result gives $X=\mathbb{C}$, whereas $\operatorname{dim}(X)=3$ implies that $X$ is an absolute sum of $\mathbb{R}$ and $\mathbb{C}$. We also give an example showing that, in general, the number of complex spaces cannot be always reduced to one. To conclude this introduction, let us mention the characterization of finite-dimensional real Hilbert spaces given by Corollary 2.7. Indeed, if $X$ is an $n$-dimensional real Banach space $X$, then we have $\operatorname{dim}(Z(X)) \leqslant \frac{n(n-1)}{2}$ (where $Z(X)$ stands for the space of those operators $T \in L(X)$ such that $\left.v(T)^{2}=0\right)$, and the inequality becomes an equality if and only if $X$ is a Hilbert space.

\section{The RESUlts}

As we commented in the introduction, real Banach spaces underlying complex Banach spaces have numerical index 0. By [15, Proposition 1 and Remark 2.a], the $c_{0^{-}}$or $\ell_{p^{-}}$sum of such a space and any other real Banach space has numerical index 0 . More generally, we also have $n(X)=0$ whenever $X=Y \oplus Z$ with $n(Z)=0$, and the direct sum is absolute (see [14]). Recall that a direct-sum $Y \oplus Z$ is said to be an absolute sum if $\|y+z\|$ only depends on $\|y\|$ and $\|z\|$ for $(y, z) \in Y \times Z$. For background on absolute sums the reader is referred to [17] and references therein. The next result somehow generalizes all these examples. We say that a real vector space has a complex structure if it is the real space underlying a complex vector space.

Proposition 2.1. Let $X$ be a real Banach space, and let $Y, Z$ be closed subspaces of $X$, with $Z \neq 0$. Suppose that $Z$ is endowed with a complex structure, that $X=Y \oplus Z$, and that the equality $\left\|y+\mathrm{e}^{i \rho} z\right\|=\|y+z\|$ holds for every $(\rho, y, z) \in$ $\mathbb{R} \times Y \times Z$. Then we have $n(X)=0$. 
Proof. First, we note that the restriction of the norm of $X$ to $Z$ converts $Z$ into a complex Banach space. Let $T$ be the nonzero operator on $X$ given by

$$
T(y, z)=i z \quad(y \in Y, z \in Z) .
$$

Let us prove that $v(T)=0$. Indeed, given $x^{*} \in S_{X^{*}}, x \in S_{X}$ with $x^{*}(x)=1$, we can write

$$
x^{*}=\left(y^{*}, \operatorname{Re} z^{*}\right), \quad x=(y, z),
$$

where $y^{*} \in Y^{*}, z^{*} \in Z^{*}, y \in Y$, and $z \in Z$. Since

$$
\left\|x^{*}\right\|=1, \quad \text { and } \quad x^{*}(x)=1, \quad \text { and } \quad\left\|y+\mathrm{e}^{i \rho} z\right\|=1 \quad(\rho \in \mathbb{R}),
$$

we have

$$
\operatorname{Re} z^{*}\left(\mathrm{e}^{i \rho} z\right) \leqslant \operatorname{Re} z^{*}(z) \quad(\rho \in \mathbb{R}) .
$$

Thus, $z^{*}(z) \in \mathbb{R}$, and hence $x^{*}(T x)=\operatorname{Re} i z^{*}(z)=0$.

It is natural to ask if every Banach space with numerical index 0 can be written in the above form. As a matter of fact, the following example shows that the numerical index 0 does not necessarily mean any kind of complex structure.

Example 2.2. There exists a (real) polyhedral Banach space $X$ with numerical index 0 . Therefore, $X$ does not contain any isometric copy of $\mathbb{C}$. Indeed, for every $k \in \mathbb{N}$, let $X_{k}$ be a 2 -dimensional real space with $n\left(X_{k}\right) \leqslant 1 / k$ and such that $B_{X_{k}}$ is a polygon. The existence of such $X_{k}$ is assured by the continuity of the numerical index with respect to equivalent norms in $\mathbb{R}^{2}$ (see [6].) Now, let $X$ be the $c_{0}$-sum of the family $\left\{X_{k}: k \in \mathbb{N}\right\}$. Then, $X$ is polyhedral (see [7, Proposition 6.11]) and $n(X)=0$ by [15, Proposition 1].

The above example has an additional interest. Indeed, since each $X_{k}$ has property $\beta$, Proposition 5 in [6] gives $n\left(X_{k}\right)>0$ for every $k \in \mathbb{N}$, and hence, as is done in [15, Examples 3.b], the numerical radius is a (non-equivalent) norm in $X$.

The aim of this paper is to prove a characterization of finite-dimensional real Banach spaces with numerical index 0 which, in particular, implies that such spaces cannot be polyhedral (compare Example 2.2). One of the facts we will use in the proof is that a finite-dimensional real Banach space with numerical index 0 contains nonzero operators whose numerical ranges reduce to $\{0\}$. A useful characterization of such operators is given by the next lemma, which follows straightforwardly from the "exponential formula" for numerical ranges [3, Theorem 3.4].

Lemma 2.3. Let $X$ be a real Banach space, and let $T$ be in $L(X)$. Then, the following are equivalent:

(i) $v(T)=0$.

(ii) $\exp (\rho T)$ is an isometry for every $\rho \in \mathbb{R}$.

We can now state the main result of the paper.

Theorem 2.4. Let $(X,\|\cdot\|)$ be a finite-dimensional real Banach space. Then, the following are equivalent:

(i) The numerical index of $X$ is zero.

(ii) There are nonzero complex vector spaces $X_{1}, \ldots, X_{n}$, a real vector space $X_{0}$, and positive integer numbers $q_{1}, \ldots, q_{n}$ such that $X=X_{0} \oplus X_{1} \oplus \cdots \oplus X_{n}$ and

$$
\left\|x_{0}+\mathrm{e}^{i q_{1} \rho} x_{1}+\cdots+\mathrm{e}^{i q_{n} \rho} x_{n}\right\|=\left\|x_{0}+x_{1}+\cdots+x_{n}\right\|
$$




$$
\text { for all } \rho \in \mathbb{R}, x_{j} \in X_{j}(j=0,1, \ldots, n) \text {. }
$$

Proof. $(i) \Rightarrow(i i)$. The finite dimensionality of $X$, together with the assumption (i), implies the existence of $T \in L(X) \backslash\{0\}$ with $v(T)=0$. On the other hand, the finite dimensionality of $X$ allows us to apply Auerbach's Theorem (see [20, Theorem 9.5.1]) assuring the existence of an inner product $(\cdot \mid \cdot)$ on $X$ such that every linear isometry on $X$ remains an isometry on $H:=(X,(\cdot \mid \cdot))$. It follows from Lemma 2.3 that $(T x \mid x)=0$ for every $x \in S_{H}$. Thus, the symmetric bilinear form on $H$ given by

$$
(x, y) \longmapsto(T x \mid y)+(x \mid T y)
$$

is zero, and hence we have $T^{*}=-T$ in $L(H)$. Let $\alpha_{1}, \ldots, \alpha_{m} \in \mathbb{R}^{+}$be such that the nonzero roots of the characteristic polynomial of $T$ are $\pm i \alpha_{1}, \ldots, \pm i \alpha_{m}$. Since $T^{2}$ is a self-adjoint operator on $H$ whose nonzero eigenvalues are $-\alpha_{1}^{2}, \ldots,-\alpha_{m}^{2}$, we have $X=Y_{0} \oplus Y_{1} \oplus \cdots \oplus Y_{m}$, where $Y_{0}:=\operatorname{ker}\left(T^{2}\right)$ and $Y_{j}$ stands for the eigenspace of $T^{2}$ corresponding to the eigenvalue $-\alpha_{j}^{2}(j \in\{1,2, \ldots, m\})$. Since the projections on $X$ corresponding to the decomposition $X=Y_{0} \oplus Y_{1} \oplus \cdots \oplus Y_{m}$ are polynomials in $T^{2}$, they commute with $T$, and hence, for $j \in\{0,1, \ldots, m\}$, the subspace $Y_{j}$ is invariant under $T$. Since for $j \in\{1,2, \ldots, m\}$ we have $T^{2}=-\alpha_{j}^{2}$ on $Y_{j}$, the space $Y_{j}$ is endowed with a complex structure by defining $i x_{j}=\alpha_{j}^{-1} T\left(x_{j}\right)$ for every $x_{j} \in Y_{j}$. Moreover, since $T^{*} T=-T^{2}$ in $L(H)$, and $Y_{0}=\operatorname{ker}\left(T^{2}\right)$, we have $Y_{0}=\operatorname{ker}(T)$. It follows that, for $\rho \in \mathbb{R}$ and $\left(x_{0}, x_{1}, \ldots, x_{m}\right) \in Y_{0} \times Y_{1} \times \cdots \times Y_{m}$, the equality

$$
\exp (\rho T)\left(x_{0}+x_{1}+\cdots+x_{m}\right)=x_{0}+\mathrm{e}^{i \alpha_{1} \rho} x_{1}+\cdots+\mathrm{e}^{i \alpha_{m} \rho} x_{m}
$$

holds, and therefore, by Lemma 2.3, we have

$$
\left\|x_{0}+\mathrm{e}^{i \alpha_{1} \rho} x_{1}+\cdots+\mathrm{e}^{i \alpha_{m} \rho} x_{m}\right\|=\left\|x_{0}+x_{1}+\cdots+x_{m}\right\| .
$$

If $\left\{\alpha_{1}, \ldots, \alpha_{m}\right\} \subseteq \mathbb{Q}$, then, taking $N \in \mathbb{N}$ such that $q_{j}=N \alpha_{j} \in \mathbb{N}$ for every $j \in\{1, \ldots, m\}$, and putting $X_{j}:=Y_{j}$ for $j=0,1, \ldots, m$, we are done.

Suppose otherwise that not every $\alpha_{k}$ is rational. Up to a rearrangement, we may find $r \in \mathbb{N}, 1 \leqslant r \leqslant m$, satisfying that $\left\{1, \alpha_{1}, \ldots, \alpha_{r}\right\}$ is $\mathbb{Q}$-linearly independent and that, when $r<m$, there exist $s_{j}, s_{k, j} \in \mathbb{Q}$ for every $k=1, \ldots, r$ and every $j=r+1, \ldots, m$, such that

$$
\alpha_{j}=s_{j}+\sum_{k=1}^{r} s_{k, j} \alpha_{k} \quad(j=r+1, \ldots, m) .
$$

We notice that, in the case that $r$ is equal to either 1 or $m$, the arguments which follow have natural simplifications. We choose $N \in \mathbb{N}$ such that

$$
N s_{r, j} \in \mathbb{Z}, \quad N s_{j} \in \mathbb{Z} \quad(j=r+1, \ldots, m)
$$

and we take

$$
n=m-r+1, \quad q_{1}=N, \quad \text { and } q_{\ell}=N s_{r, r+\ell-1} \quad(\ell=2, \ldots, n) .
$$

Let us fix

$$
x_{0}+x_{1}+\cdots+x_{m} \in X=Y_{0} \oplus Y_{1} \oplus \cdots \oplus Y_{m},
$$

and define $f: \mathbb{R} \longrightarrow \mathbb{R}$ by

$$
f(\rho)=\left\|\left(x_{0}+\cdots+x_{r-1}\right)+\mathrm{e}^{i q_{1} \rho} x_{r}+\cdots+\mathrm{e}^{i q_{n} \rho} x_{m}\right\| \quad(\rho \in \mathbb{R}) .
$$


Our aim now is to prove that $f$ is constant, which clearly implies $(i i)$ by just taking $X_{0}=Y_{0} \oplus \cdots \oplus Y_{r-1}, X_{\ell}=Y_{r+\ell-1}$ for $\ell=1, \ldots, n$ (if some $q_{\ell}$ is negative, then change the complex product in $X_{\ell}$ by its complex conjugate). We put

$$
K:=\max \left\{\left|N s_{k, j}\right|: k=1, \ldots r ; j=r+1, \ldots, m\right\} .
$$

Let us fix $t \in \mathbb{R}$ and $\varepsilon>0$. By Kronecker's Approximation Theorem [9, Theorem 442], there exist $M \in \mathbb{N}$ and $p_{1}, \ldots, p_{r} \in \mathbb{Z}$ such that

$$
\begin{aligned}
\mid t-\left(2 M \pi \alpha_{r}-2 \pi p_{r}\right) & <\varepsilon \\
\left|2 M \pi \alpha_{k}-2 \pi p_{k}\right| & <\varepsilon \quad(k=1, \ldots, r-1) .
\end{aligned}
$$

Therefore,

$$
\begin{aligned}
\left|\exp \left(i t q_{1}\right)-\exp \left(i 2 M N \pi \alpha_{r}\right)\right| & <N \varepsilon \\
\left|1-\exp \left(i 2 M N \pi \alpha_{k}\right)\right| & <N \varepsilon \quad(k=1, \ldots, r-1) .
\end{aligned}
$$

By using (2) it is not hard to check that

$$
\left|\exp \left(i t q_{\ell}\right)-\exp \left(i 2 M N \pi \alpha_{r+\ell-1}\right)\right|<r K \varepsilon \quad(\ell=2, \ldots, n) .
$$

Indeed, since $p_{k}, N s_{r+\ell-1}, N s_{k, r+\ell-1} \in \mathbb{Z}$ for $k=1, \ldots, r$ and $\ell=2, \ldots, n$, we easily get that

$$
\begin{aligned}
& \left|\exp \left(i t q_{\ell}\right)-\exp \left(i 2 M N \pi \alpha_{r+\ell-1}\right)\right| \\
& \quad=\left|\exp \left(i t N s_{r, r+\ell-1}\right)-\exp \left(i \sum_{k=1}^{r}\left(2 M N \pi s_{k, r+\ell-1} \alpha_{k}-2 N \pi s_{k, r+\ell-1} p_{k}\right)\right)\right| \\
& \quad \leqslant\left|t N s_{r, r+\ell-1}-\sum_{k=1}^{r}\left(2 M N \pi s_{k, r+\ell-1} \alpha_{k}-2 N \pi s_{k, r+\ell-1} p_{k}\right)\right| \\
& \quad \leqslant\left|N s_{r, r+\ell-1}\right|\left|t-\left(2 M \pi \alpha_{r}-2 \pi p_{r}\right)\right|+\sum_{k=1}^{r-1}\left|N s_{k, r+\ell-1}\right|\left|2 M \pi \alpha_{k}-2 \pi p_{k}\right| \\
& \quad<K \varepsilon+(r-1) K \varepsilon,
\end{aligned}
$$

where the last inequality holds by (2). Now, applying (1) for $\rho=2 M N \pi$, we have that

$$
f(0)=\left\|x_{0}+\mathrm{e}^{i 2 M N \pi \alpha_{1}} x_{1}+\cdots+\mathrm{e}^{i 2 M N \pi \alpha_{m}} x_{m}\right\|,
$$

so we deduce from (3) and (4) that

$$
|f(t)-f(0)| \leqslant N\left(\left\|x_{1}\right\|+\cdots+\left\|x_{r}\right\|\right) \varepsilon+r K\left(\left\|x_{r+1}\right\|+\cdots+\left\|x_{m}\right\|\right) \varepsilon .
$$

Letting $\varepsilon \rightarrow 0$, we get $f(t)=f(0)$ as desired.

$$
\begin{aligned}
& (i i) \Rightarrow(i) \text {. Let } T \in L(X) \text { be the nonzero operator defined by } \\
& T\left(x_{0}+x_{1}+\cdots+x_{n}\right)=i q_{1} x_{1}+\cdots+i q_{n} x_{n} \quad\left(x_{j} \in X_{j}, \quad j=0,1, \ldots, n\right) .
\end{aligned}
$$

Now, (ii) can be read as $\exp (\rho T)$ is an isometry for every $\rho \in \mathbb{R}$.

In dimension two or three, the above result can be written in the more suitable form given by Corollary 2.5 which follows. We note that, for a direct proof of such a corollary, it is enough to stop at Equation (1) in the proof of Theorem 2.4, and, consequently, the application of Kronecker's Approximation Theorem is avoidable.

Corollary 2.5. Let $X$ be a real Banach space with numerical index 0 . 
(a) If $\operatorname{dim}(X)=2$, then $X$ is isometrically isomorphic to the two-dimensional real Hilbert space.

(b) If $\operatorname{dim}(X)=3$, then $X$ is an absolute sum of $\mathbb{R}$ and the two-dimensional real Hilbert space.

Proof. (a) is clear. To realize (b), observe that the only possibility is $X_{0}=\mathbb{R}$, $n=1, X_{1}=\mathbb{C}$. Also, from

$$
\left\|\lambda+\mathrm{e}^{i t} z\right\|=\|\lambda+z\| \quad\left(t \in \mathbb{R}, \lambda \in X_{0}, z \in X_{1}\right),
$$

it is clear that $\|\lambda+z\|$ depends only on $|\lambda|$ and $|z|$.

Remark 2.6. One may wonder if, analogously to (a) in the above corollary, the unique two-dimensional complex space with numerical index $1 / 2$ is the Hilbert space. But, this is not the case. Indeed, in [5, Theorem 3.5] (or [4, §32]), the reader can find a family of two-dimensional complex spaces whose numerical indices fill the whole interval $\left[\mathrm{e}^{-1}, 1\right]$. Going into the proof, one realizes that all the spaces in the family contain segments in the unit sphere. Therefore, none of them is a Hilbert space.

For every real Banach space $X$, let us denote by $Z(X)$ the subspace of $L(X)$ consisting of those operators $T$ on $X$ with $v(T)=0$.

Corollary 2.7. Let $X$ be a real Banach space of dimension $n \in \mathbb{N}$. Then we have $\operatorname{dim}(Z(X)) \leqslant \frac{n(n-1)}{2}$. Moreover, $X$ is a Hilbert space if and only if $\operatorname{dim}(Z(X))=$ $\frac{n(n-1)}{2}$.

Proof. First, we note that, if $X$ is a Hilbert space, then we have

$$
Z(X)=\left\{T \in L(X): T^{*}=-T\right\}
$$

and hence $\operatorname{dim}(Z(X))=\frac{n(n-1)}{2}$. In the general case, we can argue as in the beginning of the proof of Theorem 2.4 to find an inner product $(\cdot \mid \cdot)$ on $X$ such that, putting $H:=(X,(\cdot \mid \cdot))$, we have the inclusion $Z(X) \subseteq Z(H)$, and therefore the inequality $\operatorname{dim}(Z(X)) \leqslant \frac{n(n-1)}{2}$ holds. Assume that $\operatorname{dim}(Z(X))=\frac{n(n-1)}{2}$. Then we must have the equality $Z(X)=Z(H)$. Let $Y$ be a two-dimensional subspace of $X$. Take a basis $\{u, v\}$ of $Y$. Then the operator $T$ on $X$ defined by $T(x):=(x \mid u) v-(x \mid v) u$ satisfies $T^{*}=-T$ in $L(H)$, and hence lies in $Z(X)$. Moreover, since the range of $T$ is contained in $Y$, the subspace $Y$ is invariant under $T$. It follows that, restricting $T$ to $Y$, we are provided with a nonzero operator on $Y$ whose numerical radius is zero. By Corollary 2.5.(a), $Y$ is a Hilbert space.

In view of Corollary 2.5 it might be thought that the number of complex spaces in Assertion ( $i i$ ) of Theorem 2.4 can be always reduced to one or, equivalently, that there are no finite-dimensional real Banach spaces with numerical index zero others than those given by Proposition 2.1. As a matter of fact, this is not true, as the following example shows.

Example 2.8. There exists a four-dimensional real space $X$ with $n(X)=0$ and such that the number of complex spaces in Theorem 2.4.(ii) cannot be reduced to one. Let $X=\mathbb{R}^{4}$ with norm

$$
\|(a, b, c, d)\|=\frac{1}{4} \int_{0}^{2 \pi}\left|\operatorname{Re}\left(\mathrm{e}^{2 i t}(a+i b)+\mathrm{e}^{i t}(c+i d)\right)\right| d t \quad(a, b, c, d \in \mathbb{R})
$$


let us write $\|\cdot\|^{*}$ for the dual norm of $\|\cdot\|$. We write

$$
X_{1}=\{(a, b, 0,0): a, b \in \mathbb{R}\}, \quad X_{2}=\{(0,0, c, d): c, d \in \mathbb{R}\},
$$

$P_{1}$ for the projection with range $X_{1}$ and kernel $X_{2}$, and $P_{2}=I d-P_{1}$. It is straightforward to check that $X_{1}, X_{2}$ are complex normed spaces (identifying $(a, b, 0,0)=$ $a+i b$ and $(0,0, c, d)=c+i d)$ and $(i i)$ in Theorem 2.4 is satisfied for $q_{1}=2, q_{2}=1$, that is,

$$
\left\|\mathrm{e}^{i 2 \rho}(a, b, 0,0)+\mathrm{e}^{i \rho}(0,0, c, d)\right\|=\|(a, b, c, d)\| \quad(\rho \in \mathbb{R}, a, b, c, d \in \mathbb{R}) .
$$

In particular, we have $n(X)=0$ and, more precisely, $v(T)=0$, where $T \in L(X)$ is given by the matrix

$$
T \equiv\left(\begin{array}{cccc}
0 & 2 & 0 & 0 \\
-2 & 0 & 0 & 0 \\
0 & 0 & 0 & 1 \\
0 & 0 & -1 & 0
\end{array}\right)
$$

(see the proof of $(i i) \Rightarrow(i)$, or use directly Lemma 2.3$)$. Observe finally that, using (5) for $\rho=\pi$, we get $\left\|2 P_{1}-I d\right\|=1$, which yields $\left\|P_{1}\right\|=\left\|P_{2}\right\|=1$, and so

$$
\|(s, t, 0,0)\|^{*}=\sqrt{s^{2}+t^{2}}, \quad\|(0,0, s, t)\|^{*}=\sqrt{s^{2}+t^{2}} \quad(s, t \in \mathbb{R}) .
$$

Now we are going to prove that the unique operators on $X$ with numerical radius 0 are the multiples of $T$, which implies that the unique possible decomposition of $X$ as in Theorem 2.4.(ii) is $X=X_{1} \oplus X_{2}$ with $q_{1}=2 q_{2}$. Let $S \in L(X)$ with $v(S)=0$, and let $\left(s_{i j}\right)$ be the matrix representation of $S$. Since

$$
\|(1,0,0,0)\|^{*}=\|(1,0,0,0)\|=\langle(1,0,0,0),(1,0,0,0)\rangle=1,
$$

we have $s_{11}=0$ and, analogously, $s_{22}=s_{33}=s_{44}=0$. From

$$
\left\|\left(\frac{1}{\sqrt{2}}, \frac{1}{\sqrt{2}}, 0,0\right)\right\|^{*}=\left\|\left(\frac{1}{\sqrt{2}}, \frac{1}{\sqrt{2}}, 0,0\right)\right\|=\left\langle\left(\frac{1}{\sqrt{2}}, \frac{1}{\sqrt{2}}, 0,0\right),\left(\frac{1}{\sqrt{2}}, \frac{1}{\sqrt{2}}, 0,0\right)\right\rangle=1,
$$

we get $s_{12}+s_{21}=0$ and, similarly, $s_{34}+s_{43}=0$. We also have that

$$
\|(0,0,1,0)\|^{*}=\|(0,1 / 2,1,0)\|=\langle(0,0,1,0),(0,1 / 2,1,0)\rangle=1,
$$

which implies $s_{32}=0$ and, analogously, $s_{42}=0$. Therefore, we have

$$
S \equiv\left(\begin{array}{cccc}
0 & s_{12} & s_{13} & s_{14} \\
-s_{12} & 0 & s_{23} & s_{24} \\
s_{31} & 0 & 0 & s_{34} \\
s_{41} & 0 & -s_{34} & 0
\end{array}\right)
$$

Applying the above to

$$
U=T S-S T \equiv\left(\begin{array}{cccc}
0 & 0 & s_{14}+2 s_{23} & -s_{13}+2 s_{24} \\
0 & 0 & -2 s_{13}+s_{24} & -2 s_{14}-s_{23} \\
s_{41} & -2 s_{31} & 0 & 0 \\
-s_{31} & -2 s_{41} & 0 & 0
\end{array}\right)
$$

(which has numerical radius zero by [19, Lemma 2.2] or by arguing as in the proof of [3, Lemma 5.4]), we get $s_{31}=s_{41}=0$. But then, $U$ is an operator on $X$ with $v(U)=0$ and such that 0 is the unique root of its characteristic polynomial. 
Applying Auerbach's theorem as in the beginning of the proof of Theorem 2.4, we get $U=0$, which yields $s_{13}=s_{14}=s_{23}=s_{24}=0$. Therefore,

$$
S \equiv\left(\begin{array}{cccc}
0 & \alpha & 0 & 0 \\
-\alpha & 0 & 0 & 0 \\
0 & 0 & 0 & \beta \\
0 & 0 & -\beta & 0
\end{array}\right)
$$

for suitable $\alpha, \beta \in \mathbb{R}$. Now, the space $Z(X)$ (of those operators $S \in L(X)$ with $v(S)=0$ ) has dimension less or equal than 2 by $(6)$. Let us show that $\operatorname{dim}(Z(X))=$ 1 (that is, each $S \in Z(X)$ is a multiple of $T$ ). Indeed, $\operatorname{dim}(Z(X)) \geqslant 1$ since $T \in Z(X)$. If $\operatorname{dim}(Z(X))=2$, then the operator $S$ given by (6) for $\alpha=\beta=1$ has numerical radius 0 , which implies $\exp (\rho S)$ to be an isometry for every $\rho \in \mathbb{R}$. But this is not the case, since

$$
\|(1,0,1,0)\|=3 \sqrt{3} / 4, \quad\left\|\exp \left(\frac{\pi}{2} S\right)(1,0,1,0)\right\|=\|(0,1,0,1)\|=5 / 4 .
$$

Therefore, every operator on $X$ with numerical radius 0 is a multiple of $T$, as desired.

Acknowledgement. The authors wish to express their gratitude to the referee for several useful suggestions which improved the final form of the paper.

\section{REFERENCES}

[1] F. L. Bauer, On the field of values subordinate to a norm, Numer. Math., 4 (1962), $103-111$.

[2] H. F. Bohnenblust and S. Karlin, Geometrical properties of the unit sphere in Banach algebras, Ann. of Math., 62 (1955), 217-229.

[3] F. F. Bonsall and J. Duncan, Numerical Ranges of operators on normed spaces and of elements of normed algebras, London Math. Soc. Lecture Note Ser. 2, Cambridge, 1971.

[4] F. F. Bonsall and J. Duncan, Numerical Ranges II, London Math. Soc. Lecture Note Ser. 10, Cambridge, 1973.

[5] J. Duncan, C. M. McGregor, J. D. Pryce, and A. J. White, The numerical index of a normed space, J. London Math. Soc. 2 (1970), 481-488.

[6] C. Finet, M. Martín, and R. Payá, Numerical index and renorming, Proc. Amer. Math. Soc. 131 (2003), 871-877.

[7] V. P. Fonf, J. Lindenstrauss, and R. R. Phelps, Infinite dimensional convexity. Handbook of the geometry of Banach spaces, Vol. I, 599-670, North-Holland, Amsterdam, 2001.

[8] B. W. Glickfeld, On an inequality of Banach algebra geometry and semi-inner-product space theory, Illinois J. Math., 14 (1970), 76-81.

[9] G. H. Hardy and E. M. Wright, An introduction to the theory of numbers (5th edition), Clarendon Press, Oxford, 1979.

[10] A. Kaidi, A. Morales, and A. Rodríguez-Palacios, Geometrical properties of the product of a $C^{*}$-algebra, Rocky Mountain J. Math. 31 (2001), 197-213.

[11] G. López, M. Martín, and R. Payá, Real Banach spaces with numerical index 1, Bull. London Math. Soc. 31 (1999), 207-212.

[12] G. Lumer, Semi-inner-product spaces, Trans. Amer. Math. Soc. 109 (1961), 29-43.

[13] M. Martín, A survey on the numerical index of a Banach space, Extracta Math. 15 (2000), $265-275$.

[14] M. Martín, Numerical index and subspaces (Spanish), Proceedings of the Meeting of Andalusian Mathematicians, Vol. II (Spanish) (Sevilla, 2000), 641-648, Colecc. Abierta, 52, Univ. Sevilla Secr. Publ., Seville, 2001.

[15] M. Martín and R. Payá, Numerical index of vector-valued function spaces, Studia Math. 142 (2000), 269-280.

[16] M. Martín and A. R. Villena, Numerical index and Daugavet property for $L_{\infty}(\mu, X)$, Proc. Edinburgh Math. Soc. 46 (2003), 415-420. 
[17] J. F. Mena, R. Payá, A. Rodríguez-Palacios, and D. Yost, Absolutely proximinal subspaces of Banach spaces, J. Aprox. Theory 65 (1991), 46-72.

[18] T. Oikhberg, Spaces of operators, the $\psi$-Daugavet property, and numerical indices, to appear.

[19] A. Rodríguez-Palacios, An approach to Jordan-Banach algebras from the theory of Nonassociative complete normed algebras, Ann. Sci. Univ. Clermont-Ferrand II Math. No. 27 (1991), $1-57$.

[20] S. Rolewicz, Metric Linear Spaces (Second edition), Mathematics and its Applications (East European Series), 20. D. Reidel Publishing Co., Dordrecht; PWN—Polish Scientific Publishers, Warsaw, 1985.

[21] O. Toeplitz, Das algebraische Analogon zu einem Satze von Fejer, Math. Z.2 (1918), 187-197.

Departamento de Análisis Matemático, Facultad de Ciencias, Universidad de Granada, 18071 Granada, Spain, e-mail : mmartins@ugr.es

Departamento de AnÁlisis Matemático, Facultad de Ciencias, Universidad de Granada, 18071 Granada, Spain, e-mail : jmeri@ugr.es

Departamento de Análisis Matemático, Facultad de Ciencias, Universidad de Granada, 18071 Granada, Spain, e-mail : apalacio@ugr.es 